\title{
Multiscale Modeling of Biopolymer Production in Multicellular Systems
}

\author{
A. Franz ${ }^{*}$, H. Grammel ${ }^{* *}$, R. Rehner***, P. Paetzold ${ }^{* * *}$, A. Kienle ${ }^{* * * * *}$ \\ * Process Synthesis and Process Dynamics, Max Planck Institute, \\ Magdeburg, Germany (email: afranz@mpi-magdeburg.mpg.de) \\ ** Systems Biology, Max Planck Institute, Magdeburg, Germany (email: \\ grammel@mpi-magdeburg.mpg.de) \\ *** Systems Biology, Max Planck Institute, Magdeburg, Germany \\ (email: ruxandra.rehner@mpi-magdeburg.mpg.de) \\ **** Process Synthesis and Process Dynamics, Max Planck Institute, \\ Magdeburg, Germany (email: paetzold@mpi-magdeburg.mpg.de) \\ ***** Chair of Automation/Modeling, Otto-von-Guericke Universität, \\ Magdeburg, Germany / Process Synthesis and Process Dynamics, Max \\ Planck Institute, Magdeburg, Germany (email: \\ kienle@mpi-magdeburg.mpg.de)
}

\begin{abstract}
In this contribution we present a multiscale modeling approach to systematically explore the heterogeneity of biopolymer production in multicellular systems. The first step is a dynamic single cell model which is based on the hybrid cybernetic modeling approach to include cell internal regulation. The single cell model is used for nonlinear analysis and the occurrence of multiple steady states is discussed. The single cell model is then reduced by using the lumped hybrid cybernetic approach and by approximation of enzyme levels. The reduced single cell model is used to develop a population balance model for predicting heterogeneity in multicellular production processes. Thus, we have combined the cybernetic modeling approach with population balance modeling, which consequently includes cell internal regulation.

The multiscale modeling approach is first developed for the microorganism Ralstonia eutropha, which serves as a model and benchmark organisms. Good agreement between model predictions and experimental data is shown. An extension to Rhodospirillum rubrum is discussed, which shows interesting transient bimodal dynamics.
\end{abstract}

Keywords: Biopolymer, Cybernetic modeling, Population balance modeling, Bistability, Multiscale modeling

\section{INTRODUCTION}

Poly( $\beta$-hydroxybutyrate) (PHB) is an organic polymer, which can be synthesized by many microorganisms and which serves as internal energy and carbon reserve material. PHB provides an attractive source of bioplastics that are biodegradable, biocompatible and do not depend on fossil resources. The production of PHB is favored under limitation of key nutrients such as nitrogen, phosphate or oxygen and can be degraded if these nutrients are available.

There are three main processes, namely: growth, PHB synthesis and PHB degradation, between the organisms will switch depending on available substrates and internal metabolite concentration. Switching between these processes is controlled by cell internal regulation and can lead to bi- or even multistability on the single cell level. Hence, a dynamic model has to include appropriate cell internal regulation. This regulation is considered by using the cybernetic modeling approach. In the cybernetic approach optimal regulation in view of available resources is assumed. Based on this approach a population balance model is developed, which considers cell internal regulation by means of cybernetic control variables. Since bi- and multistability on the single cell level can translate to biand multimodality on the population level, it is necessary to include cell internal regulation into population balance modeling.

Experimental findings for Rhodospirillum rubrum (see Figure 1) shows interesting bimodal behavior and indicate bistability on the single cell level. The multiscale modeling approach can be used to explore the source of bistability/bimodality and can therefore help to gain a better understanding of this nonlinear behavior and to improve industrial processes for PHB synthesis.

\section{MATERIAL AND METHODS}

\subsection{Ralstonia eutropha}

For cultivation conditions and analytical procedures for Ralstonia eutropha see Franz et al. (2011). 

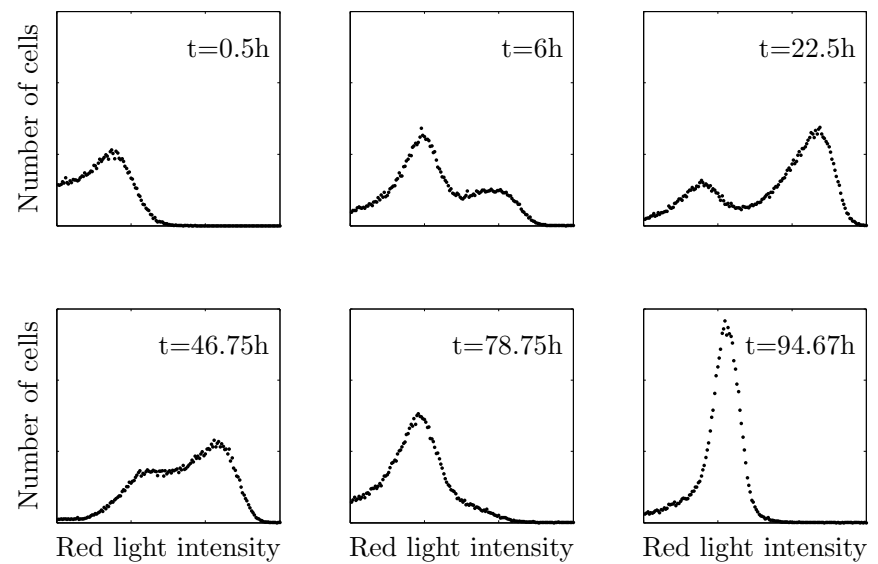

Fig. 1. Experimental data from flow cytometry with Rhodospirillum rubrum. Cells were stained with nile red and red light intensity corresponds to PHB content.

\subsection{Rhodospirillum rubrum}

Rhodospirillum rubrum strain S1 (ATCC 11170) was grown aerobically in $0.5 \mathrm{~L}$ baffled shake-flasks containing $100 \mathrm{~mL}$ mineral medium and incubated on a rotary shaker (Certomat BS1, Sartorius, Goettingen, Germany) at $30^{\circ} \mathrm{C}$ and 100rpm.

The mineral medium used for cultivation contained (per $\mathrm{L}): 0.82 \mathrm{~g} \mathrm{CH}_{3} \mathrm{COONa}, 1.48 \mathrm{~g} \mathrm{~K}_{2} \mathrm{HPO}_{4}, 2.22 \mathrm{~g} \mathrm{KH}_{2} \mathrm{PO}_{4}$, $0.43 \mathrm{~g} \mathrm{NH} \mathrm{NH}_{4} \mathrm{Cl}, 0.12 \mathrm{~g} \mathrm{NaCl}, 2.85 \mathrm{mg}$ p-aminobenzoic acid, $0.2 \mathrm{~g}$ nitrilotriacetic acid, 40mg l-aspartate, 100mg lglutamate, $250 \mathrm{mg} \mathrm{KOH}, 589.8 \mathrm{mg} \mathrm{MgSO}_{4} \times 7 \mathrm{H}_{2} \mathrm{O}$, $3 \mathrm{mg}$ $\mathrm{FeSO}_{4} \times 7 \mathrm{H}_{2} \mathrm{O}, 1 \mathrm{mg}$ nicotinic acid, $0.5 \mathrm{mg}$ thiamine hydrochloride, $0.02 \mathrm{mg}$ biotin, $66 \mathrm{mg} \mathrm{CaCl}_{2} \times 2 \mathrm{H}_{2} \mathrm{O}, 10 \mathrm{mg}$ EDTA, 4.4mg ZnSO $4 \times \mathrm{H}_{2} \mathrm{O}, 2.3 \mathrm{mg} \mathrm{H}_{3} \mathrm{BO}_{3}, 1 \mathrm{mg} \mathrm{MnCl}_{3}$ $\times 4 \mathrm{H}_{2} \mathrm{O}, 0.32 \mathrm{mg} \mathrm{CoCl} \mathrm{Cl}_{2} \times 6 \mathrm{H}_{2} \mathrm{O}, 0.22 \mathrm{mg} \mathrm{CuSO}_{4} \times 5 \mathrm{H}_{2} \mathrm{O}$, $0.22 \mathrm{mg} 2\left(\mathrm{NH}_{4}\right)_{6} \mathrm{Mo}_{7} \mathrm{O}_{24} \times \mathrm{H}_{2} \mathrm{O}$.

Optical density (OD) was determined at 660nm using a $\mathrm{UV}$-vis spectrophotometer.

\subsection{Sample preparation for flow cytometry}

Samples were diluted with $\mathrm{NaCl}(0.98 \%(\mathrm{w} / \mathrm{v}))$ to $\mathrm{OD}$ 0.1 . $1 \mathrm{ml}$ of diluted samples were centrifuged in $1.5 \mathrm{~mL}$ Eppendorf cups at 13000rpm. Supernatant was removed carefully and the remaining pellets were dissolved in $40 \mu \mathrm{L}$ PBS and $6 \mu \mathrm{L}$ Nile red $(100 \mathrm{~g} / \mathrm{L})$ was added. Samples were then incubated in a thermo shaker for $15 \mathrm{~min}$ at $37^{\circ} \mathrm{C}$ and $450 \mathrm{rpm}$. For flow cytometry $2 \mu \mathrm{L}$ of the sample were then dissolved in $2 \mathrm{~mL}$ PBS.

\section{SINGLE CELL MODEL}

In a first step single cell kinetics are described by a state of the art hybrid cybernetic model (HCM) (see Kim et al. (2008); Song et al. (2009)). The HCM allows a systematic derivation of the model equations from elementary mode analysis (see Stelling et al. (2002)) and has a moderate complexity, which is useful for nonlinear analysis. The $\mathrm{HCM}$ is based on quasistationarity of internal metabolites, which are eliminated from the model equations. However $\mathrm{PHB}$ is an internal metabolite. Hence, the HCM approach was extended to take dynamics of few internal metabolites explicitly into account, while for most of the internal metabolites the quasi-steady state approximation is still applied (see Franz et al. (2011)).

The model equations are given by

$$
\begin{aligned}
\frac{\mathrm{d}}{\mathrm{d} t}\left[\begin{array}{c}
x_{\mathrm{FRU}} \\
x_{\mathrm{AMC}}
\end{array}\right] & =D\left(\left[\begin{array}{c}
x_{\mathrm{FRU}}^{\mathrm{in}} \\
x_{\mathrm{AMC}}^{\mathrm{in}}
\end{array}\right]-\left[\begin{array}{c}
x_{\mathrm{FRU}} \\
x_{\mathrm{AMC}}
\end{array}\right]\right)+\mathbf{S}_{\mathbf{s}} \mathbf{Z} \mathbf{r}_{\mathbf{M}} c \\
\frac{\mathrm{d}}{\mathrm{d} t} m_{\mathrm{PHB}} & =\mathbf{S}_{\mathbf{m}, \mathbf{s}} \mathbf{Z}_{\mathbf{M}}-\mu m_{\mathrm{PHB}} \\
\frac{\mathrm{d} \mathbf{e}}{\mathrm{d} t} & =\boldsymbol{\alpha}+\mathbf{r}_{\mathbf{E M}} b-\operatorname{diag}(\boldsymbol{\beta}) \mathbf{e}-\mu \mathbf{e} \\
\frac{\mathrm{d} c}{\mathrm{~d} t} & =(\mu-D) c
\end{aligned}
$$

where $x_{\mathrm{FRU}}$ and $x_{\mathrm{AMC}}$ are the concentration of the cell external substrates fructose as carbon source and ammonium chloride as nitrogen source, $\mathbf{S}_{\mathbf{s}} \mathbf{Z}$ and $\mathbf{S}_{\mathbf{m}, \mathbf{s}} \mathbf{Z}$ are stoichiometric matrices, which contain the elementary mode data, $m_{\mathrm{PHB}}$ is the specific $\mathrm{PHB}$ concentration, $c$ is the concentration of total biomass (TBM = non-PHB biomass + PHB) and $\mathbf{e}$ is the vector of relative enzyme levels, which catalyze the five active elementary modes determined by metabolic yield analysis (see Song and Ramkrishna (2009)). The fluxes through elementary modes $\mathbf{r}_{\mathbf{M}}$ and enzyme synthesis rates $\mathbf{r}_{\mathbf{E M}}$ are controlled by the cybernetic control variables $\mathbf{u}$ and $\mathbf{v}$ respectively

$$
\begin{aligned}
\mathbf{r}_{\mathbf{M}} & =\operatorname{diag}(\mathbf{v}) \operatorname{diag}(\mathbf{e}) \operatorname{diag}\left(\mathbf{k}_{\mathbf{r}}\right) \mathbf{r}^{\text {core }} \\
\mathbf{r}_{\mathbf{E M}} & =\operatorname{diag}(\mathbf{u}) \operatorname{diag}\left(\mathbf{k}_{\mathbf{e}}\right) \mathbf{r}^{\text {core }}
\end{aligned}
$$

where the core rates are Monod type

$$
\begin{aligned}
& r_{1}^{\text {core }}=\frac{x_{\mathrm{FRU}}}{K_{\mathrm{FRU}}+x_{\mathrm{FRU}}} \\
& r_{i}^{\text {core }}=\frac{x_{\mathrm{FRU}}}{K_{\mathrm{FRU}}+x_{\mathrm{FRU}}} \frac{x_{\mathrm{AMC}}}{K_{\mathrm{AMC}}+x_{\mathrm{AMC}}}, \quad i=2,3,4 \\
& r_{5}^{\text {core }}=\frac{x_{\mathrm{FRU}}}{K_{\mathrm{FRU}}+x_{\mathrm{FRU}}} \frac{x_{\mathrm{AMC}}}{K_{\mathrm{AMC}}+x_{\mathrm{AMC}}} \frac{m_{\mathrm{PHB}}}{K_{\mathrm{PHB}}+m_{\mathrm{PHB}}}
\end{aligned}
$$

The cybernetic control laws are given by

$$
\mathbf{u}=\frac{\mathbf{p}}{\|\mathbf{p}\|_{1}}, \quad \mathbf{v}=\frac{\mathbf{p}}{\|\mathbf{p}\|_{\infty}}
$$

where $\mathbf{p}$ is the return on investment (ROI), which can be calculated from a metabolic objective function. In this study it is assumed that the organism maximizes carbon source uptake and $\mathbf{p}$ is therefore defined as:

$$
\mathbf{p}=\operatorname{diag}\left(\mathbf{f}_{\mathbf{c}}\right) \operatorname{diag}(\mathbf{e}) \operatorname{diag}\left(\mathbf{k}_{\mathbf{r}}\right) \mathbf{r}^{\text {core }}
$$

where $\mathbf{f}_{\mathbf{c}}$ is the vector of uptaken carbon units.

The vector of rate constants $\mathbf{k}_{\mathbf{r}}$ was estimated by fitting the model equations to a set of experimental data (see Figure 2), where all three main processes were stimulated separately.

Model predictions show very good agreement with additional independent data sets (data sets II-IV in Figure 3). 
Data set I:
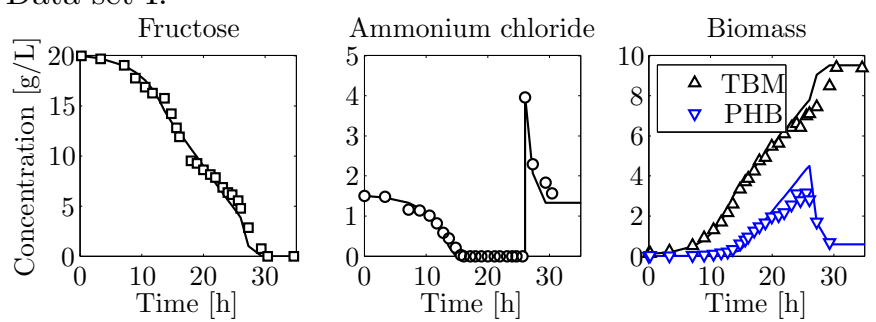

Fig. 2. Hybrid cybernetic model $(-)$ and experimental data $(\square, \bigcirc, \nabla, \triangle)$. Data set I was used for parameter identification. Substrates were fructose as carbon source and ammonium chloride $\left(\mathrm{NH}_{4} \mathrm{Cl}\right)$ as nitrogen source. Biomass concentration is represented by total biomass (TBM) and PHB.

Data set II:
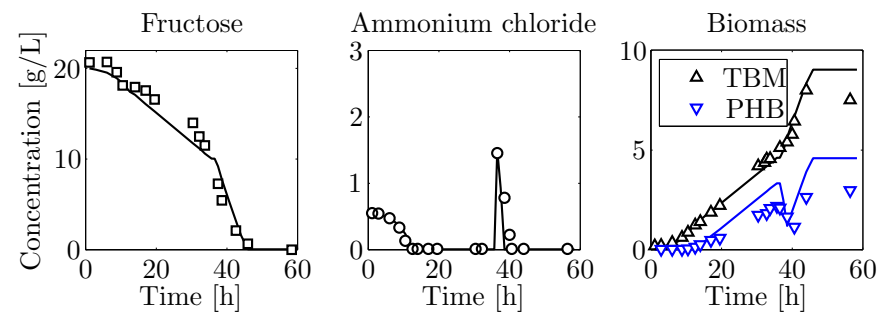

Data set III:
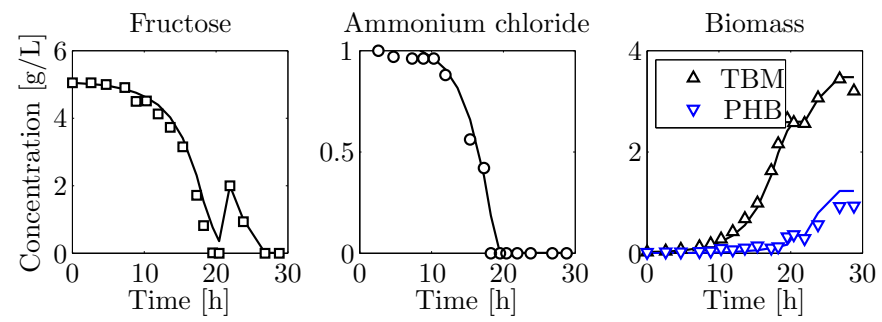

Data set VI:
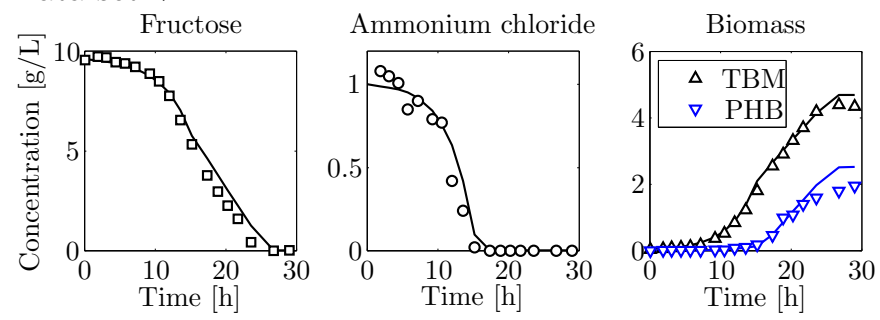

Fig. 3. Independent data sets $(\square, \bigcirc, \nabla, \triangle)$ show very good agreement with model prediction $(-)$.

\subsection{Nonlinear analysis}

The model was then used to investigate the possibility of bistability on the single cell level. The bistability region for Ralstonia eutropha predicted by the model is rather small (see Figure 4) and will vanish for high $\gamma=x_{\mathrm{AMC}}^{\text {in }} / x_{\mathrm{FRU}}^{\mathrm{in}}$ (data not shown). It is therefore argued that multiple steady states are unlikely to occur in practice for this specific system. This is confirmed by flow cytometry experiments which show only unimodal distribution (see Figure 6) compared to experiments with Rhodospirillum rubrum which show bimodal distributions (see Figure 1).

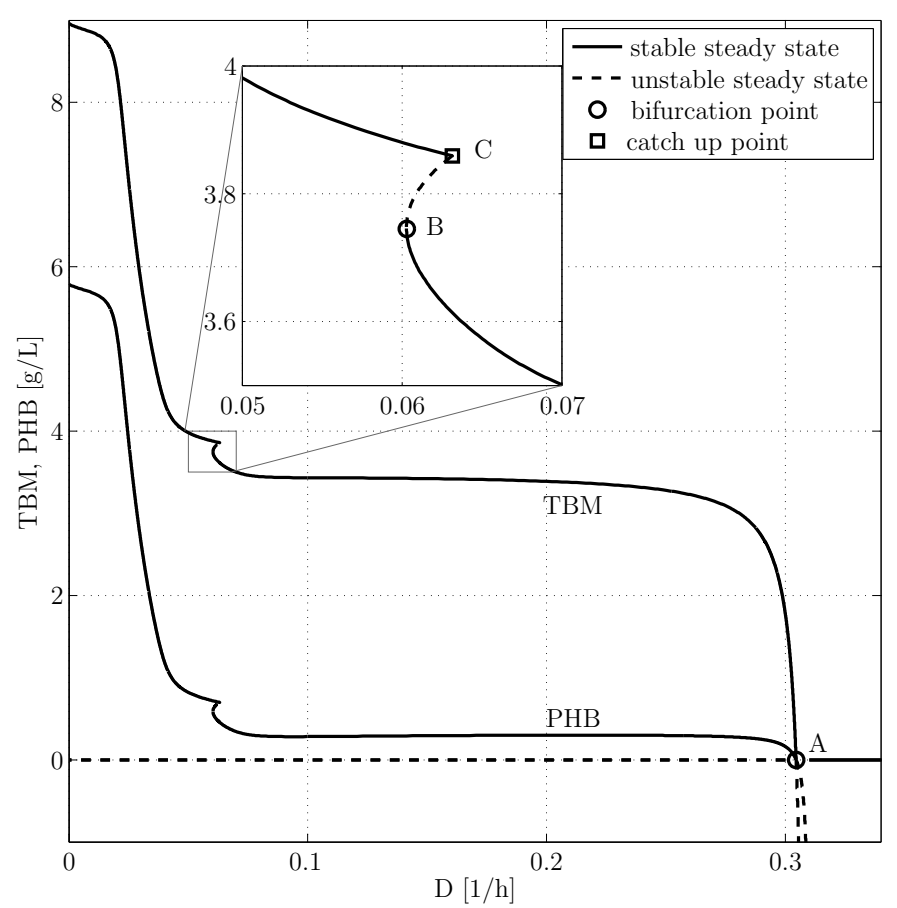

Fig. 4. Bifurcation study for Ralstonia eutropha. The predicted multiplicity region is rather small and it is argued that multiple steady states are therefore unlikely to occur in practice for this specific system.

\subsection{Reduction of model dimension}

The developed HCM consists of nine ODEs: five ODEs for the key enzymes of elementary modes, two ODEs for the internal metabolite concentration of non-PHB biomass and PHB and two ODEs for the cell external substrate concentrations of fructose and ammonium chloride. The internal metabolite concentrations and enzyme levels will translate into internal coordinates in population balance equation. The resulting PBM will therefore have seven internal coordinates, which is quite challenging for numerical solution. Therefore the model has to be reduced.

In a first step a lumped hybrid cybernetic model (LHCM) approach (see Song and Ramkrishna (2010, 2011)) is used, where elementary modes are lumped, for instance according to their ATP requirements. The five elementary modes in the HCM can be lumped into three lumped elementary modes, since for each main process at least one lumped elementary mode is necessary. However, PHB consumption can also be neglected in a first step. Hence, the developed L-HCM consists of two lumped elementary modes and therefore of six state variables, where four of them (two enzyme level and two internal metabolite concentration) will translate into internal coordinates of the PBE.

Since the enzyme level dynamics are very fast in this specific system, enzyme levels can be assumed to be at quasi steady state. In this case the enzyme level in equation (5) can be approximated by $\mathbf{e}=\mathbf{u}$ (see Baloo and Ramkrishna (1991) and Young and Ramkrishna (2007)). The internal coordinates for the enzyme levels in PBE can then be omitted and the resulting PBE has only two internal coordinates, namely non-PHB biomass and PHB. 
In general, if enzyme level dynamics are fast enough and can be assumed to be in quasi steady state, these can be omitted as internal coordinates in PBE. In this case even a HCM with a huge number of elementary modes can be reduced significantly, without using L-HCM. In the case, where enzyme levels can not be assumed to be in quasi steady state, the number of internal coordinates in PBE can only be reduced by reducing the number of elementary modes, e.g. by lumping elementary modes. However, this is only possible up to a certain degree, since for every main process at least one lumped elementary mode is necessary.

Figure 5 shows the comparison of the HCM, HCM with approximated enzyme levels, L-HCM, L-HCM with approximated enzyme levels and experimental data sets III and IV. All models are in good agreement with experimental data. Data sets I and II are not used for model comparison, since these data sets include PHB consumption, which is neglected in the L-HCM.

Data set III:
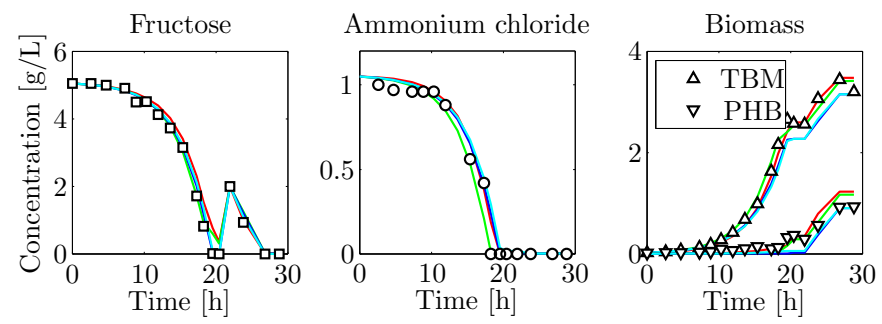

Data set IV:
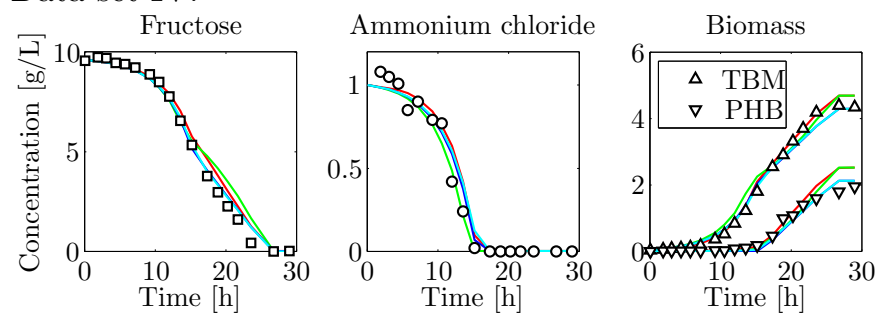

Fig. 5. Comparison of HCM (red), HCM with approximated enzyme levels (green), L-HCM (blue), L-HCM with approximated enzyme levels (cyan) and experimental data $(\square, \bigcirc, \nabla, \triangle)$.

Hence, the reduced model can be used for further investigation.

\section{POPULATION BALANCE MODEL}

Based on the L-HCM with approximated enzyme levels a 2D population balance model (PBM) is developed, which considers cell internal regulation. The rates, respectively fluxes through space of internal coordinates are controlled by cybernetic control variables. Depending on available substrates and position in space of internal coordinates the PBM is therefore able to switch between growth and PHB synthesis.

$$
\begin{array}{r}
\frac{\partial N\left(\mathbf{x}_{\mathbf{c}}, t\right)}{\partial t}+\frac{\partial}{\partial x_{\mathrm{BIO}}}\left(r^{\mathrm{BIO}} N\left(\mathbf{x}_{\mathbf{c}}, t\right)\right) \\
+\frac{\partial}{\partial x_{\mathrm{PHB}}}\left(r^{\mathrm{PHB}} N\left(\mathbf{x}_{\mathbf{c}}, t\right)\right) \\
\left.=2 \int_{\mathbf{x}_{\mathbf{c}}} \int_{\mathbf{c}, \max }^{\mathbf{x}_{\mathbf{c}}}, \mathbf{x}_{\mathbf{s}}\right) p\left(\mathbf{x}_{\mathbf{c}}, \mathbf{x}_{\mathbf{c}}^{*}, \mathbf{x}_{\mathbf{s}}\right) N\left(\mathbf{x}_{\mathbf{c}}^{*}, t\right) \mathrm{d} \mathbf{x}_{\mathbf{c}}^{*} \\
-\Gamma\left(\mathbf{x}_{\mathbf{c}}, \mathbf{x}_{\mathbf{s}}\right) N\left(\mathbf{x}_{\mathbf{c}}, t\right)
\end{array}
$$

with $\mathbf{x}_{\mathbf{s}}=\left[x_{\mathrm{FRU}} x_{\mathrm{AMC}}\right]^{\mathrm{T}}$ and $\mathbf{x}_{\mathbf{c}}=\left[x_{\mathrm{BIO}} m_{\mathrm{PHB}}\right]^{\mathrm{T}}$, where $x_{\mathrm{BIO}}$ is the concentration of non-PHB biomass. The fluxes in direction of non-PHB biomass synthesis $r^{\mathrm{BIO}}$ and $\mathrm{PHB}$ synthesis $r^{\mathrm{PHB}}$ are regulated by the cybernetic control variables. For low ammonium chloride concentration and high fructose concentration synthesis of PHB is favored, while non-PHB biomass is increasing when there is sufficient ammonium chloride available.

Cell division occurs Gaussian distributed around a certain amount of non-PHB biomass $\bar{x}_{\mathrm{BIO}}$ and is independent of $\mathrm{PHB}$ concentration and it is assumed that cells divide into two equal daughter cells.

$$
\Gamma\left(\mathbf{x}_{\mathbf{c}}, \mathbf{x}_{\mathbf{s}}\right)=\frac{f\left(x_{\mathrm{BIO}}\right)}{1-\int f\left(x_{\mathrm{BIO}}\right) \mathrm{d} x_{\mathrm{BIO}}} r^{\mathrm{BIO}}\left(\mathbf{x}_{\mathbf{c}}, \mathbf{x}_{\mathbf{s}}\right)
$$

with

$$
f\left(x_{\mathrm{BIO}}\right)=\frac{1}{\sigma \sqrt{2 \pi}} \exp \left(-\frac{\left(x_{\mathrm{BIO}}-\bar{x}_{\mathrm{BIO}}\right)^{2}}{2 \sigma^{2}}\right)
$$

For the numerical solution a finite volume approach is applied.
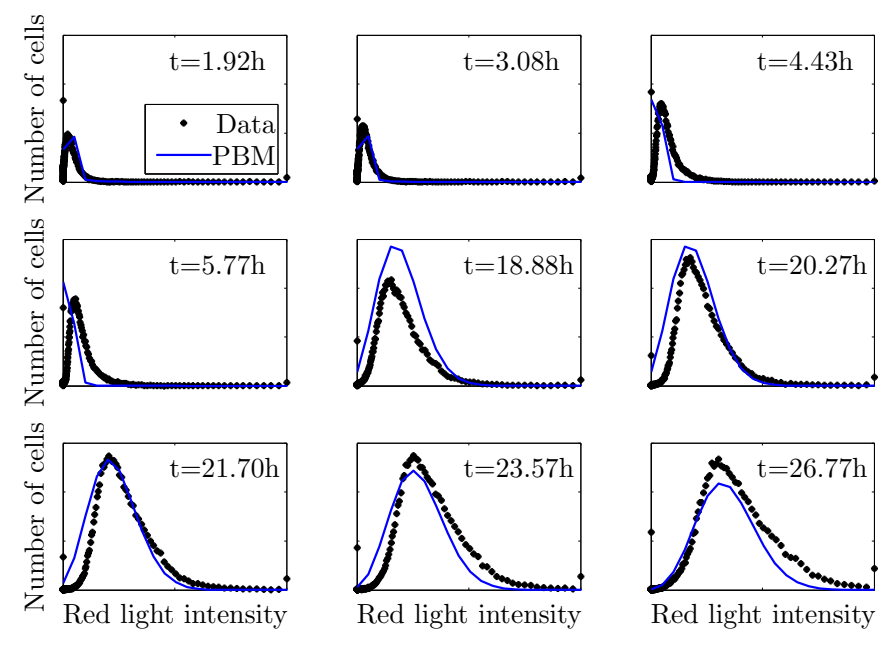

Fig. 6. Unimodal distribution for Ralstonia eutropha. Data from flow cytometry (dotted, black) and model prediction (solid, blue). Cells were stained with nile red and red light intensity corresponds to PHB content.

Figure 6 shows, that the model predicts a unimodal distribution which is in agreement with theoretical analysis with single cell kinetics in section 3.1 and experimental data measured by flow cytometry for Ralstonia eutropha. 


\section{CONCLUSION AND FUTURE WORK}

In this study a multiscale modeling approach was presented. This approach can be used to systematically explore heterogeneity/homogeneity of biopolymer production in multicellular systems.

At first a single cell model was developed, which accounts for cell internal regulation. The single cell model was used for nonlinear analysis. Based on the single cell model a population balance model was developed which also accounts for cell internal regulation. Since state variables of the single cell model will translate into internal coordinates of the population balance model, the single cell model was first reduced by lumping elementary modes and approximation of enzyme levels.

Application of the multiscale modeling approach to Ralstonia eutropha shows a homogeneous distribution which is in good agreement with experimental data.

Currently the multiscale modeling approach is applied to Rhodospirillum rubrum, which shows a heterogeneous distribution (see Figure 1).

\section{ACKNOWLEDGEMENTS}

We appreciate the financial support of the German Federal Ministry for Education and Research (BMBF) under the FORSYS program.

\section{REFERENCES}

Baloo, S. and Ramkrishna, D. (1991). Metabolic regulation in bacterial continuous cultures: II. Biotechnology and Bioengineering, 38(11), 1353-1363.

Franz, A., Song, H.S., Ramkrishna, D., and Kienle, A. (2011). Experimental and theoretical analysis of poly(beta-hydroxybutyrate) formation and consumption in ralstonia eutropha. Biochemical Engineering Journal, 55(1), 49-58.

Kim, J.I., Varner, J.D., and Ramkrishna, D. (2008). A hybrid model of anaerobic E. coli GJT001: combination of elementary flux modes and cybernetic variables. Biotechnol. Prog., 24(5), 993-1006.

Song, H.S., Morgan, J.A., and Ramkrishna, D. (2009). Systematic development of hybrid cybernetic models: application to recombinant yeast co-consuming glucose and xylose. Biotechnol. Bioeng., 103(5), 984-1002.

Song, H.S. and Ramkrishna, D. (2009). Reduction of a set of elementary modes using yield analysis. Biotechnol. Bioeng., 102(2), 554-568.

Song, H.S. and Ramkrishna, D. (2010). Prediction of metabolic function from limited data: Lumped hybrid cybernetic modeling (L-HCM). Biotechnol. Bioeng., 106(2), 271-284.

Song, H.S. and Ramkrishna, D. (2011). Cybernetic models based on lumped elementary modes accurately predict strain-specific metabolic function. Biotechnol. Bioeng., 108(1), 127-140.

Stelling, J., Klamt, S., Bettenbrock, K., Schuster, S., and Gilles, E.D. (2002). Metabolic network structure determines key aspects of functionality and regulation. Nature, 420(6912), 190-193.
Young, J.D. and Ramkrishna, D. (2007). On the matching and proportional laws of cybernetic models. Biotechnol. Prog., 23(1), 83-99.

\section{Appendix A. NOMENCLATURE}

$D$

e vector of relative enzyme levels

$\mathbf{f}_{\mathbf{c}} \quad$ vector of uptaken carbon units

$\mathbf{k}_{\mathbf{e}} \quad$ vector of enzyme synthesis rate constants

$\mathbf{k}_{\mathbf{r}} \quad$ vector of rate constants

$K_{\mathrm{AMC}}$ saturation constant for ammonium chloride

$K_{\text {FRU }}$ saturation constant for fructose

$K_{\mathrm{PHB}}$ saturation constant for $\mathrm{PHB}$

$m_{\mathrm{PHB}} \quad$ specific concentration of $\mathrm{PHB}$

$p$

p

$r^{\mathrm{PHB}}$

$\mathrm{r}_{\mathrm{EM}}$

partition probability density function

vector of ROI

flux in direction of non-PHB biomass synthesis

flux in direction of PHB synthesis

vector of regulated enzyme synthesis rates of EMs

$\mathbf{r}_{\mathbf{M}} \quad$ vector of regulated fluxes through EMs

$\mathbf{r}_{\mathbf{M}}$ core vector of core rates

$\mathbf{S}_{\mathbf{m}, \mathbf{s}} \mathbf{Z}$ stoichiometry matrix

$\mathbf{S}_{\mathbf{s}} \mathbf{Z} \quad$ stoichiometry matrix

$\mathbf{u}$ vector of cybernetic variables which control enzyme synthesis

v vector of cybernetic variables which control enzyme activity

$x_{\mathrm{AMC}}$ concentration of ammonium chloride

$x_{\mathrm{FRU}} \quad$ concentration of fructose

$x_{\mathrm{AMC}}^{\text {in }}$ concentration of ammonium chloride at inlet

$x_{\mathrm{FRU}}^{\mathrm{in}}$ concentration of fructose at inlet

$\mathbf{x}_{\mathbf{c}} \quad$ state vector of biomass concentration

$\mathbf{x}_{\mathbf{s}} \quad$ state vector of substrate concentration

$\boldsymbol{\alpha} \quad$ vector of constitutive enzyme synthesis rates

$\boldsymbol{\beta} \quad$ vector of enzyme consumption constants

$\gamma \quad$ feed composition

$\mu \quad$ growth rate

$\Gamma \quad$ division rate

\section{Appendix B. ABBREVIATIONS}

HCM Hybrid cybernetic model

L-HCM Lumped hybrid cybernetic model

ROI Return on investment

PBE Population balance equation

PBM Population balance modeling

PHB Poly $(\beta$-hydroxybutyrate)

TBM Total biomass (cell dry weight) 